\title{
Alternative Transfers to the NEOs 99942 Apophis, 1994 WR12, and 2007 UW1 via Derived Trajectories from Periodic Orbits of Family G
}

\author{
C. F. de Melo, ${ }^{1}$ E. E. N. Macau, ${ }^{2}$ and O. C. Winter ${ }^{3}$ \\ ${ }^{1}$ UFABC, Universidade Federal do ABC, Santo André, 09210-170, SP, Brazil \\ ${ }^{2}$ INPE, Instituto Nacional de Pesquisas Espaciais, São José dos Campos, 12227-010, SP, Brazil \\ ${ }^{3}$ UNESP, Universidade Estadual Paulista, Guaratinguetá, 12516-410, SP, Brazil \\ Correspondence should be addressed to C. F. de Melo, cristianofiorilo@terra.com.br
}

Received 30 July 2009; Accepted 11 December 2009

Recommended by Maria F. P. S. Zanardi

\begin{abstract}
Swing-by techniques are extensively used in interplanetary missions to minimize fuel consumption and to raise payloads of spacecrafts. The effectiveness of this type of maneuver has been proven since the beginning of space exploration. According to this premise, we have explored the existence of a natural and direct link between low Earth orbits and the lunar sphere of influence to get low-energy transfer trajectories to the Near Earth Objects (NEOs) 99942 Apophis, 1994 WR12, and 2007 UW1 through swing-bys with the Moon. The existence of this link is related to a family of retrograde periodic orbits around the Lagrangian equilibrium point L1 predicted for the circular, planar, restricted three-body Earth-Moon-particle problem. The trajectories in this link are sensitive to small disturbances. This enables them to be conveniently diverted reducing so the cost of the swing-by maneuver. These maneuvers allow a gain in energy sufficient for the trajectories to escape from the Earth-Moon system and to stabilize in heliocentric orbits between the Earth and Venus or Earth and Mars. Therefore, the trajectories have sufficient reach to intercept the NEOs' orbits.
\end{abstract}

Copyright (C) 2009 C. F. de Melo et al. This is an open access article distributed under the Creative Commons Attribution License, which permits unrestricted use, distribution, and reproduction in any medium, provided the original work is properly cited.

\section{Introduction}

The dynamics of the circular restricted three-body Earth-Moon-particle problem predicts the existence of the retrograde periodic orbits around the Lagrangian equilibrium point L1. Such orbits belong to the so-called Family G [1], and starting from them it is possible to define a set of trajectories that form a natural round trip link between the Earth and Moon and a link between the Earth, the Moon and the distant space of the Earth sphere of influence. These links occur even for more complex dynamical systems as the complete Sun-Earth-Moonparticle problem. In the last dynamical system, many kinds of transfers can be exploited. 
For example, the round trip link is useful for transfers between Low Earth Orbits (LEOs) and Low Lunar Orbits (LLOs), including polar LLOs, since some of its trajectories pass a few hundreds of kilometers from the Earth's surface and a few dozens of kilometers from the Moon's surface (Figure 1) [2]. Moreover, the round trip link is also useful for transfer between two LEOs with different altitudes and inclinations [3]. In these cases the Moon's gravitational field, which is near the Earth, works as an extra impulse and provides an efficient method to minimize the fuel consumption to be used in plane change maneuvers. This is possible, because the trajectories of the links can lead spacecrafts in a natural way to accomplish swingbys with the Moon. In this paper, we use the link between the Earth, the Moon and the distant space of the Earth sphere of influence and the energy gain of the swing-by with the Moon to get escape trajectories and to design missions to NEOs.

In general, a swing-by, or a flyby, consists of the alteration of small celestial bodies orbits (comets, asteroids, or spacecrafts) when they have a close approach with a planet or a moon. Literature presents many studies about this subject with general descriptions, applications in interplanetary missions and in the Earth-Moon System. For example, Lawden [4], Minovitch [5], and Broucke [6] present general descriptions of the mechanics of gravityassisted maneuvers. Successful examples in interplanetary missions were the Voyager 1 and 2 which accomplished several swing-bys with the visited planets to gain energy [7], and Ulysses solar probe which accomplished a swing-by with Jupiter to get a perpendicular orbit to the ecliptic and observe the Sun's Poles [8]. Among the many studies on swing-bys in the Earth-Moon system, we can highlight Dunham and Davis [9], who designed missions with multiple swing-bys with the Moon; Uphoff [10], who gave a description about lunar gravityassisted maneuvers, and Prado [11], who presented a study to use flyby with the Moon to accomplish transfers between Earth orbits with the same altitudes and different inclinations. Among the missions that accomplished swing-bys with the Moon, we should mention the ISEE-3 which after completing its mission in 1982 was renamed ICE (International Cometary Explorer) and accomplished several lunar encounters to gain energy and intercept the comets Giacobini-Zinner in September 1985 and Halley, in March 1986 [12]. On the other hand, also in general, the literature does not present works focused on getting the trajectory that will accomplish the swing-by. In this work we do not only use the swing-by to obtain transfer trajectories, but we have included a study that involves the use of trajectories derived from periodic orbits to accomplish the swing-by in an efficient way. This allows the reduction of the swing-by maneuver costs.

Our results, considering trajectories derived from periodic orbits of Family $\mathrm{G}$ and the quasiperiodic orbits that oscillate around them, have allowed us to present the parameters to design Earth-NEOs transfers. The trajectories studied in this work can offer a saving up to $4 \%$ in relation to the conventional methods used to send spacecrafts to these asteroids.

This article is laid out according to the following order: in Section 2, the dynamical systems and the orbits of Family G are described; in Section 3, the set of trajectories of interest are defined. In Section 4, the studies about the mechanism of the proposed transfers between Earth and NEOs are shown and, in Section 5, we present and discuss the results. The conclusions on the work are presented in Section 6.

\section{Earth-Moon Link}

The equations of motion of a small particle of negligible mass, $m$, moving under the gravitational influence of two bodies with preponderant masses, written in a normalized 
rotating coordinate system $(x, y)$, also-called synodic system, are

$$
\ddot{x}-2 n \dot{y}=\frac{\partial U}{\partial x}, \quad \ddot{y}+2 n \dot{x}=\frac{\partial U}{\partial y},
$$

where $n$ is the mean motion and $U$ is a scalar function given by

$$
U=\frac{n^{2}}{2}\left(x^{2}+y^{2}\right)+\frac{\mu_{1}}{r_{1}}+\frac{\mu_{2}}{r_{2}}
$$

$\mu_{1}$ and $\mu_{2}$ are the reduced masses of the two preponderant bodies, called primaries. $r_{1}^{2}=$ $\left(x+\mu_{2}\right)^{2}+y^{2}$ and $r_{2}^{2}=\left(x-\mu_{1}\right)^{2}+y^{2}$ are the distances between the primaries and $m$. Equation (2.1) define the well-known restricted three-body problem in which the primaries have circular orbits around their common centre of mass $[1,13]$.

Equation (2.1) presents a special number of solutions that can be achieved searching for points in synodic frame where $\ddot{x}=\ddot{y}=\dot{x}=\dot{y}=0$. There are five points with these features and they are called of Lagrangiam equilibrium points, $\mathrm{L}_{i}, i=1, \ldots, 5$. The locations of these points are obtained solving the simultaneous nonlinear equations $\partial U / \partial x=\partial U / \partial y=0$. Figure 1 shows the locations of these points for Earth-Moon-particle problem. In this case, $\mu_{1}=\mu_{\text {Earth }}=0.9878494, \mu_{2}=\mu_{\text {Moon }}=0.0121506$, and $n=1$.

In the Earth-Moon-particle problem, groups of periodic orbits around the Earth, the Moon and the $\mathrm{L}_{i}$ points are known. We have considered a particular family of periodic orbits around L1 known as Family G [1-3]. In the normalized synodic frame, one of the groups of initial conditions that allow us to obtain the Family G orbits has the form

$$
\left(x_{0}, y_{0}, z_{0}, \dot{x}_{0}, \dot{y}_{0}, \dot{z}_{0}\right)=\left(x_{0}, 0,0,0, \dot{y}_{0}, 0\right)
$$

where $\left(-\mu_{\text {Moon }}+R_{E}^{*}\right)<x_{0}<x(\mathrm{~L} 1)$, with $R_{E}^{*}=R_{E} / 384,400 \mathrm{~km}=0.016572, R_{E}=6,370 \mathrm{~km}$ is the average radius of the Earth, $x(\mathrm{~L} 1)=0.836893$ is the abscissa of the Lagrangian equilibrium point L1, and $-9.389476 \leq \dot{y}_{0} \leq 601.045381$ [1], which corresponds to a variation between $-9.607 \mathrm{~km} / \mathrm{s}$ and $614.964 \mathrm{~km} / \mathrm{s}$. The average Earth-Moon distance $(384,400 \mathrm{~km})$ is chosen as the unit of length of the normalized system. According to (2.3), for any orbit of Family G, in $t=0$, the Earth, the particle and the Moon are aligned in this order, that is, in inferior conjunction. All orbits in Family G are unstable and this feature is very important for the transfers here exploited. Figure 1 also shows a typical orbit of Family G.

Periodic orbits as the one shown in Figure 1 and the quasiperiodic orbits that oscillate around them are tangents to the Earth and Moon low orbits. In other words, they define a link between the Earth and the lunar sphere of influence. This property allied with the instability of Family G orbits allows the design of controlled swing-bys with the Moon to gain sufficient energy to escape from the Earth-Moon system [2]. Moreover, we remark that these properties allow the reduction of the swing-by maneuver costs besides providing the necessary energy to win the Earth's and the Moon's gravitational fields [3,14]. Controlled swing-bys also allow the use of this energy to obtain trajectories inclination changes [3].

In order to exploit these properties and to design more efficient and realistic EarthNEOs transfers, the Sun's gravitational field must be introduced. That is, new equations of motion must be considered, this time, taking into account the Sun, the Earth and the Moon 


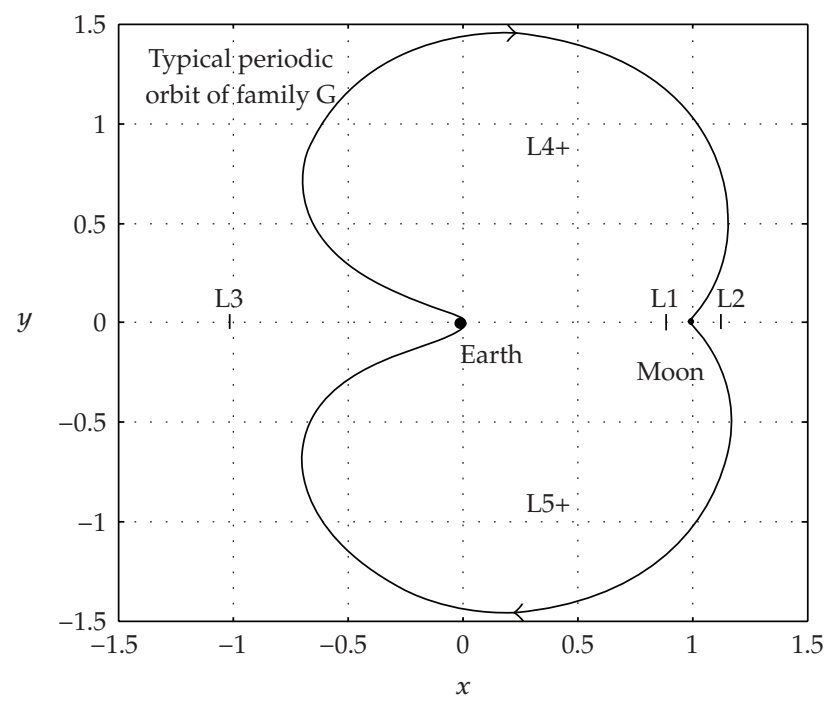

Figure 1: Locations of Lagrangian equilibrium points and a typical periodic orbit of Family $\mathrm{G}$ in synodic frame $(x, y)$.

mutual gravitational attractions, besides the characteristics of the Earth's orbit around the Sun (eccentricity) and the Moon's orbit around the Earth (eccentricity and inclination). Thus, we define the full four-body Sun-Earth-Moon-particle problem, whose 12 equations of motion in a Cartesian coordinates system $(X, Y, Z)$ with origin in a fixed point of the space are

$$
\ddot{\mathbf{R}}_{i}=\sum_{\substack{j=1 \\ j \neq i}}^{4} \frac{\mu_{j}}{R_{j i}^{3}}\left(\mathbf{R}_{j}-\mathbf{R}_{i}\right),
$$

where $R_{i j}=\left|\mathbf{R}_{j}-\mathbf{R}_{i}\right|=\left[\left(X_{j}-X_{i}\right)^{2}+\left(Y_{j}-Y_{i}\right)^{2}+\left(Z_{j}-Z_{i}\right)^{2}\right]^{1 / 2}$, with $j \neq i$, are the distances between $i$ th and $j$ th bodies. The eccentricity of the Earth's orbit and the eccentricity and inclination of the Moon's orbit are introduced through initial conditions.

The link between the Earth and the lunar sphere of influence continues existing, even when the dynamical system given by (2.4) is considered. This can be seen in Figure 2(a) that shows a trajectory found considering the full four-body problem. Figure 2(b) shows the inclination gain relative to the ecliptic after the swing-by with the Moon. Note that the trajectory inclination change is about 52 degrees. As a first conclusion, we can say that the Moon gravitational field acts like a natural propeller, without fuel consumption. In the next section, we are going to exploit this property in a controlled way. That is, taking advantage of the existing natural link between the Earth and the lunar sphere of influence to obtain a change in inclination and the energy gain needed for a determined sort of mission.

\section{Escape Trajectories}

The existence of the link between the Earth and the lunar sphere of influence for the full four-body problem is essential to the design of the Earth-Moon system escape trajectories. 


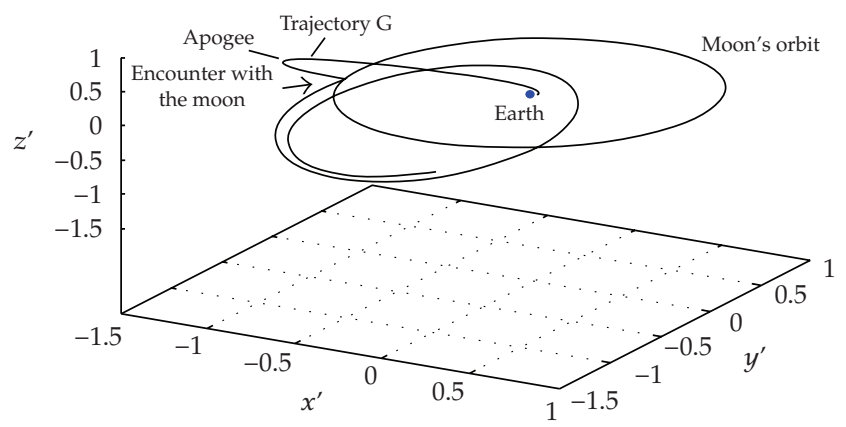

(a)

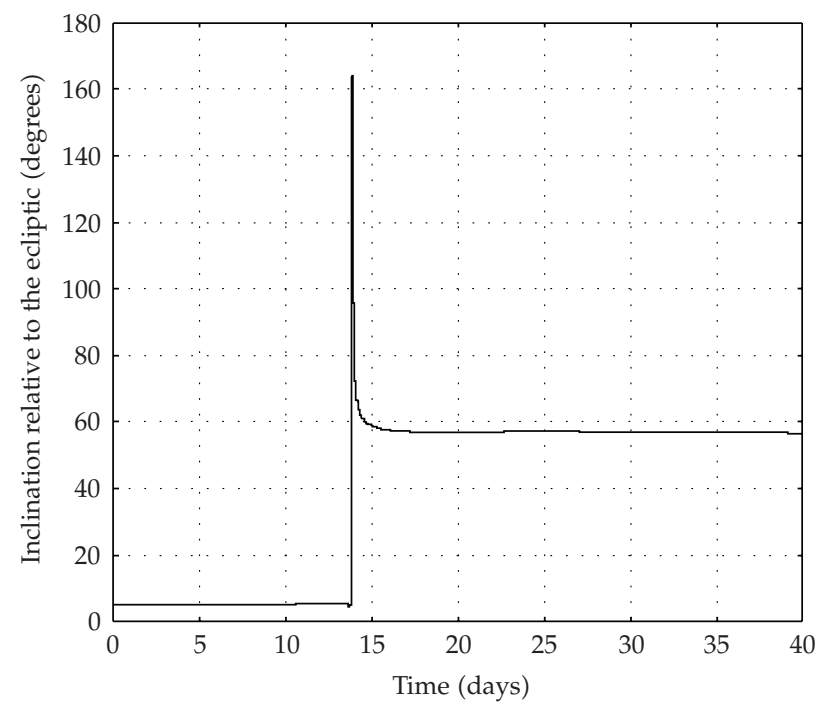

(b)

Figure 2: (a) Derived trajectory of a Family G periodic orbit considering the full four-body problem in geocentric frame $\left(x^{\prime}, y^{\prime}, z^{\prime}\right)$. (b) Trajectory inclination change as function of the time.

For this dynamical system, the initial conditions of an escape trajectory in a geocentric frame $\left(x^{\prime}, y^{\prime}, z^{\prime}\right)$ are [3]

$$
\left(x_{0}^{\prime}, y_{0}^{\prime}, z_{0}^{\prime}, \dot{x}_{0}^{\prime}, \dot{y}_{0}^{\prime}, \dot{z}_{0}^{\prime}\right)=\left(x_{0}^{\prime}, 0, z_{0}^{\prime}, 0, \dot{y}_{0}^{\prime}, 0\right)
$$

where

$$
\begin{gathered}
x_{0}^{\prime}=\left(R_{E}+h_{0}\right) \cos \left(i_{\text {Moon }}\right), \\
y_{0}^{\prime}=0, \\
z_{0}^{\prime}=\left(R_{E}+h_{0}\right) \sin \left(i_{\text {Moon }}\right), \\
\dot{x}_{0}^{\prime}=0, \\
\dot{y}_{0}^{\prime}=V_{I}, \\
\dot{z}_{0}^{\prime}=0 .
\end{gathered}
$$


Quantity $V_{I}$ is called injection velocity. Considering a circular LEO, $V_{I}=V_{\mathrm{CE}}+\Delta V_{1}$, where $V_{\mathrm{CE}}$ is the LEO's velocity, $V_{\mathrm{CE}}=\left[G M_{\mathrm{Earth}} /\left(R_{E}+h_{0}\right)\right]^{1 / 2}, G$ is the gravitational constant, $M_{\text {Earth }}$ is the Earth's mass, and $h_{0}$ is the LEO's altitude. $i_{\text {Moon }}$ is the inclination of the Moon's orbit. $\Delta V_{1}$ is the velocity increment that must be provided to the spacecraft. $\Delta V_{1}$ is a function of the $h_{0}$ and $V_{\mathrm{CE}}$ and its value is given by

$$
\Delta V_{1}=\frac{V_{C E}}{2}\left(-2.3340 \times 10^{-6} h_{0}+0.8085 \pm 0.0001\right)+\delta+\vartheta
$$

Quantity $\delta$ assumes values that will take into account the relative position among the Sun, the Earth, and the Moon. In other words, the values assumed by $\delta$ reflect the influence of the eccentricities of the Earth's orbit and, mainly, of the Moon's orbit in the distance between them. Equations to estimate the values assumed by $\delta$ as a function of the true anomalies of the Earth's and Moon's orbits are given by de Melo et al. [3].

Here, we are interested in Earth-Moon escape trajectories. Then, we can consider the Earth and the Moon in circular orbits, with the average radius of the Moon's orbit equal to $384,400 \mathrm{~km}$. This does not represent a significant loss of precision. This way, we can assume that $\delta=0$ in (3.3) and, then, we just analyze the quantity $\theta$ whose values will determine the spacecraft position relative to the Moon and the Earth during the passage by the lunar sphere of influence and the swing-by. For $-1.50 \times 10^{-4} \mathrm{~km} / \mathrm{s} \leq \vartheta \leq 1.50 \times 10^{-4} \mathrm{~km} / \mathrm{s}$, we will have collision trajectories with the Moon, for $\vartheta<-1.50 \times 10^{-4} \mathrm{~km} / \mathrm{s}$, the trajectories will have periselenium in the region close to the Moon, between itself and the Earth (anterior region). For $\vartheta>1.50 \times 10^{-4} \mathrm{~km} / \mathrm{s}$, the periselenium will be in the posterior region to the Moon relative to the Earth. For $\vartheta<-1.50 \times 10^{-4} \mathrm{~km} / \mathrm{s}$, it is possible to design transfers between LEOs and LLOs of any altitudes and also between two Earth orbits of different inclinations and altitudes, both at a low cost $[2,3]$. Our interest, however, is the trajectories generated for $v>1.50 \times 10^{-4} \mathrm{~km} / \mathrm{s}$. In those cases, the gain of energy with the swing-by guarantees the necessary velocity increment to win the Earth's and the Moon's gravitational fields.

The spacecraft must reach the velocity $\mathbf{V}_{I}$ when the Earth, the spacecraft and the Moon are aligned in this order. This is necessary because the trajectory is derived from a Family $G$ orbit, and this is the only situation in which the orbits of Family G are tangent to the circular LEOs. Note that this condition does not impose significant restrictions in practical purpose. For instance, if a satellite is in a LEO with an altitude of $200 \mathrm{~km}$, this condition will occur every 1.48 hour, approximately, which is the period of the LEO. Figure 3 shows a typical escape trajectory derived from Family $\mathrm{G}$ orbits. From now on, we will call them of escape trajectories G.

It is important to note that the trajectories evolution of the Earth-Moon and escape links shown here (Figures 2 and 3) do not suffer any intervention since they start from the LEO. That is, the inclination and the energy gain occur naturally during the passage through the lunar sphere of influence. However, it is possible to take advantage of the inherent instability of these trajectories to control the spacecraft passage through the lunar sphere of influence $[2,15]$. This idea will be exploited in the next section to generate specific escape trajectories for determined missions. 


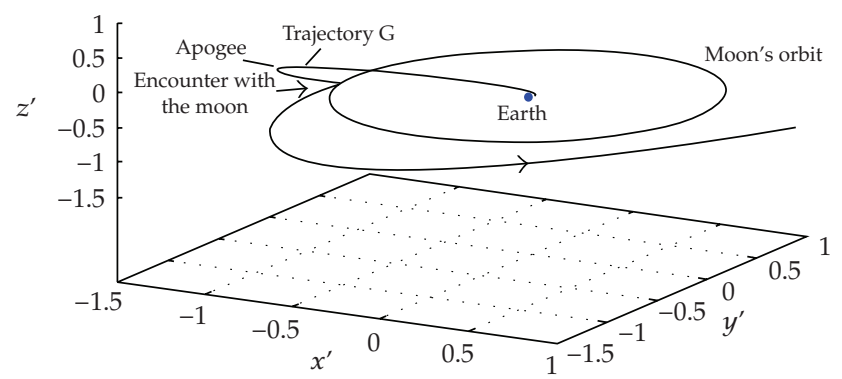

(a)

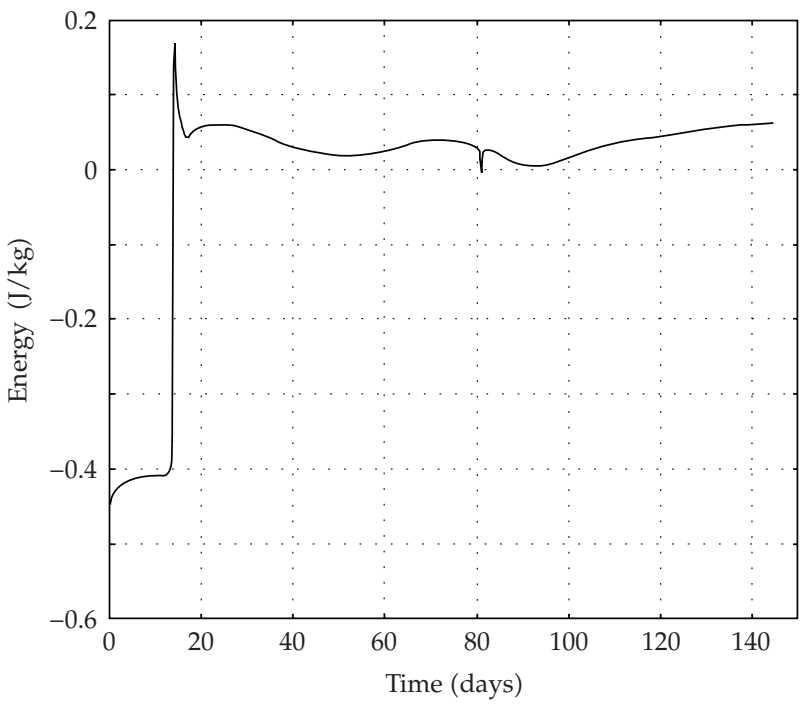

(b)

Figure 3: (a) Typical escape trajectory from the Earth-Moon system. (b) Energy gain relative to the Earth as function of the time for the trajectory. Note that it is a typical swingy-by energy gain.

\section{Escape Trajectories to Design Earth-NEOs Transfers}

The basic idea is to find transfer trajectories between the Earth and the NEOs starting from escape trajectories derived from Family G periodic orbits. This will be done to three NEOs: the 99942 Apophis, the 1994 WR12, and the 2007 UW1. All these are NEOs of the Aten asteroids class and they are defined by having semimajor axes of less than one astronomical unit ( $1 \mathrm{AU}$ is equal to $149.6 \times 10^{6} \mathrm{~km}$ ). Some data about these objects are provided in Tables 1 and 2. Table 1 shows the orbital elements: semimajor axis $(a)$, eccentricity $(e)$, inclination (i), longitude of ascending node $(\Omega)$, argument of perihelion $(w)$, and the orbital period $(P)$. Table 2 brings other information as average orbital velocity $\left(V_{\text {ave }}\right)$, Escape velocity $\left(V_{\text {esc }}\right)$, Aphelion, Perihelion, Mass, and diameter. In Table 3, the three next Closest Point Approaches (CPA) with the Earth are presented for 99942 Apophis, the 1994 WR12, and the 2007 UW1.

Once the mission target is determined, it is necessary to find the point in the asteroid's orbit where the spacecraft will reach it. However, our goal here is not to find an optimal trajectory exactly. We intend to show that transfer trajectories derived from periodic orbits around L1, generated after swing-bys with the Moon, can reach an NEO with $\Delta V \mathrm{~s}$ smaller 
Table 1: NEOs orbital elements.

\begin{tabular}{lcccccc}
\hline Asteroid & $a(\mathrm{UA})$ & $e$ & $i^{* *}(\mathrm{deg})$ & $\Omega(\mathrm{deg})$ & $w(\mathrm{deg})$ & $P(\mathrm{yr})$ \\
\hline Apophis & 0.992 & 0.191 & 3.331 & 304,5 & 126,7 & 0.89 \\
1994WR12 & 0.757 & 0.397 & 6.864 & 62,85 & 205,9 & 0.66 \\
2007UW1 & 0.907 & 0.121 & 8.224 & 26,04 & 146,5 & 0.86 \\
\hline
\end{tabular}

${ }^{* *}$ Inclination relative to the ecliptic. Source: http://neo.jpl.nasa.gov/.

Table 2: Some characteristics of the NEOs orbits.

\begin{tabular}{lcccccc}
\hline Asteroid & $V_{\text {ave }}(\mathrm{km} / \mathrm{s})$ & $V_{\text {esc }}(\mathrm{m} / \mathrm{s})$ & Aphelion $(\mathrm{UA})$ & Perihelion $(\mathrm{UA})$ & Mass $(\mathrm{kg})$ & Diameter $(\mathrm{m})$ \\
\hline Apophis & 30.728 & 0.144 & 1.099 & 0.746 & $2.7 \times 10^{10}$ & 270 \\
1994WR12 & 34.706 & 0.050 & 1.058 & 0.455 & $2.0 \times 10^{9}$ & $110-260$ \\
2007UW1 & 31.509 & 0.047 & 1.017 & 0.798 & $1.3 \times 10^{9}$ & $80-190$ \\
\hline
\end{tabular}

Source: http://neo.jpl.nasa.gov/.

than the ones required by trajectories that leave from the Earth directly. In order to do so, we only considered trajectories that are tangents to the NEOs's orbits. In order to find an escape trajectory that will reach an NEO's orbit, for example, an algorithm integrates a number of them starting from the interception point backward in time until they reach the closest point approach with the Moon during the swing-bys. These integrations are supplied for initial conditions determined starting from previous numerical simulations of trajectories that escape naturally from the Earth-Moon system, as the one shown in Figure 3. In these simulations, a group of trajectories that have a point closer to the NEO's orbit is selected. The positions and the velocities of these trajectories are analyzed and adjusted to form a group of initial conditions for the integrations beginning in the interception point of the NEO's orbit. When the closest point approach with the Moon is found, the algorithm calculates a small $\Delta \mathbf{V}$ to determine which escape trajectory $\mathrm{G}$ is more suitable for the transfer. In other words, it calculates the components values of the spacecraft's initial condition in the LEO (3.1). The algorithm provides a group of solutions with $\Delta V_{\text {Total }}, \Delta V_{1}$, and $\Delta V_{\text {intermediary }}$, transfer time and other quantities of interest. Besides the $\Delta \mathbf{V}_{\text {intermediary }}$ applied in the closest point approach with the Moon, the algorithm also calculates another $\Delta \mathbf{V}_{\text {intermediary }}$ applied in the first apogee of trajectory G. This apogee is reached about eight days after the departure from the LEO and prior to the passage by the lunar sphere of influence (Figure 3). This is the best point to do velocity and inclination changes. In this point, the trajectories $G$ velocities are very small; so small $\Delta \mathbf{V}$ s can provide significant velocity and inclination changes.

A similar procedure is used to find the transfer trajectory between the Earth and the NEO for Patched-conic approximation. In this method, the transfers are designed seeking for a heliocentric conic arc that links an LEO to an orbit around the asteroid [16].

\section{Results}

The results of the intercept missions to the NEOs Apophis, 1999 WR12, and 2007 UW1 are in Tables 4 and 5. Table 4 presents the values found for the missions conceived by escape trajectories $G$, and Table 5 presents the correspondent values found for the missions conceived by Patched-conic approach. For each sort of mission, the $\Delta V \mathrm{~s}$ applied in the departure from the LEO, for occasional velocity and inclination changes, the spacecraft velocities relative to the asteroids in the closest point approach (CPA), and the transfer times 
Table 3: Three next closest point approaches.

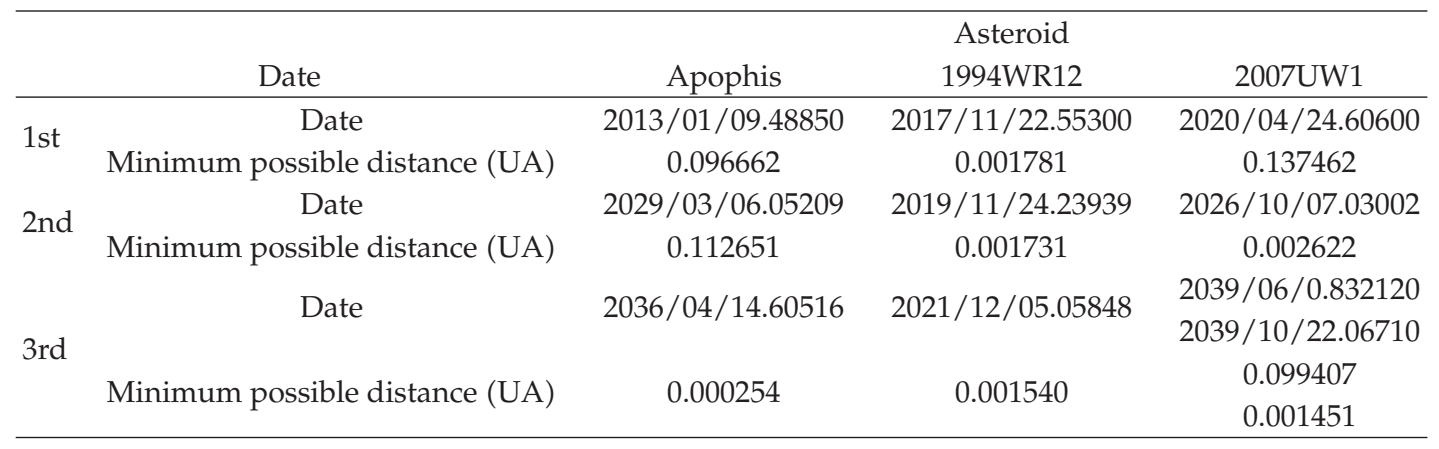

Sources: http://neo.jpl.nasa.gov/.

Table 4: Missions' data of the Earth-NEOs transfer by escape trajectories G.

\begin{tabular}{lcccccccc}
\hline Asteroid & $\begin{array}{c}\Delta V_{1}^{*} \\
(\mathrm{~km} / \mathrm{s})\end{array}$ & $\begin{array}{c}\Delta V_{\text {int1 }}^{* *} \\
(\mathrm{~km} / \mathrm{s})\end{array}$ & $\begin{array}{c}\Delta V_{\text {int2 }}^{* * *} \\
(\mathrm{~km} / \mathrm{s})\end{array}$ & $\begin{array}{c}\Delta V_{\text {Total }} \\
(\mathrm{km} / \mathrm{s})\end{array}$ & $\begin{array}{c}T 1 \\
(\text { days })\end{array}$ & $\begin{array}{c}V_{1}^{* * * *} \\
(\mathrm{~km} / \mathrm{s})\end{array}$ & $\begin{array}{c}T 2 \\
(\text { days })\end{array}$ & $\begin{array}{c}V_{2}^{* * * * *} \\
(\mathrm{~km} / \mathrm{s})\end{array}$ \\
\hline Apophis & 3.148 & 0.010 & 0.018 & 3.176 & 81 & 0.800 & 225 & 0.550 \\
1994WR12 & 3.147 & 0.018 & - & 3.165 & 53 & 3.700 & 103 & 3.600 \\
2007UW1 & 3.149 & 0.018 & 0.012 & 3.169 & 153 & 0.490 & - & - \\
\hline
\end{tabular}

${ }^{*}$ Departure, ${ }^{* *} 1$ st apogee, ${ }^{* * *} \mathrm{CPA}$ with the Moon, ${ }^{* * * *}$ spacecraft velocity relative to the asteroid in $T 1$, and ${ }^{* * * * *}$ spacecraft velocity relative to the asteroid in $T 2$.

Table 5: Missions' data of the Earth-NEOs transfer by Patched-conic approach.

\begin{tabular}{lccccccc}
\hline Asteroid & $\Delta V_{1}^{*}(\mathrm{~km} / \mathrm{s})$ & $\Delta V_{\text {int } 1}^{* *}(\mathrm{~km} / \mathrm{s})$ & $\Delta V_{\text {Total }}(\mathrm{km} / \mathrm{s})$ & $T 1$ (days) & $V_{1}^{* * *}(\mathrm{~km} / \mathrm{s})$ & $T 2$ (days) & $V_{2}^{* * * *}(\mathrm{~km} / \mathrm{s})$ \\
\hline Apophis & 3.280 & 0.020 & 3.308 & 155 & 1.200 & 198 & 1.350 \\
1994WR12 & 3.225 & 0.035 & 3.260 & 131 & 4.100 & - & - \\
2007UW1 & 3.250 & 0.028 & 3.278 & 111 & 0.690 & - & - \\
\hline
\end{tabular}

${ }^{*}$ Departure, ${ }^{* *}$ middle curse, ${ }^{* * *}$ spacecraft velocity relative to the asteroid in $T 1$, and ${ }^{* * * *}$ spacecraft velocity relative to the asteroid in $T 2$.

were calculated. The altitude of the circular departure LEO was considered equal to $200 \mathrm{~km}$, and the altitude of the CPA with the asteroids was considered equal to $1 \mathrm{~km}$. For the transfers between the Earth and Apophis and the Earth and 1994 WR12, it was possible to find two CPAs between the spacecraft and the asteroids. In Tables 4 and 5, we call these times of T1 for the 1st CPA and T2 for the 2nd CPA. It is interesting to note that spacecraft velocities relative to the asteroids are different in each CPA, and, depending on the mission goals, the choice of one of them could represent an extra fuel saving in an occasional insertion maneuver of the spacecraft into an orbit around the asteroid.

Figure 4(a) shows the Earth's and the Apophis's orbits and the heliocentric ellipse arc generated by the Patched-conic approach. The times of the 1st and 2nd CPA are 155 and 198 days after the departure from the LEO, respectively, and the distances from the Earth to these points are $11.2 \times 10^{6} \mathrm{~km}$ and $113.6 \times 10^{6} \mathrm{~km}$, respectively. The values of $T 1$ and $T 2$ of the other transfers are in Tables 4 and 5.

By analogy, Figure 4(b) shows the escape trajectory $G$ found for the Earth-Apophis transfer. The 1st and the 2nd CPAs take place 86 and 225 days after the departure from the $\mathrm{LEO}$, and the distances from the Earth to these points are $52.27 \times 10^{6} \mathrm{~km}$ and $204.45 \times 10^{6} \mathrm{~km}$, respectively. 


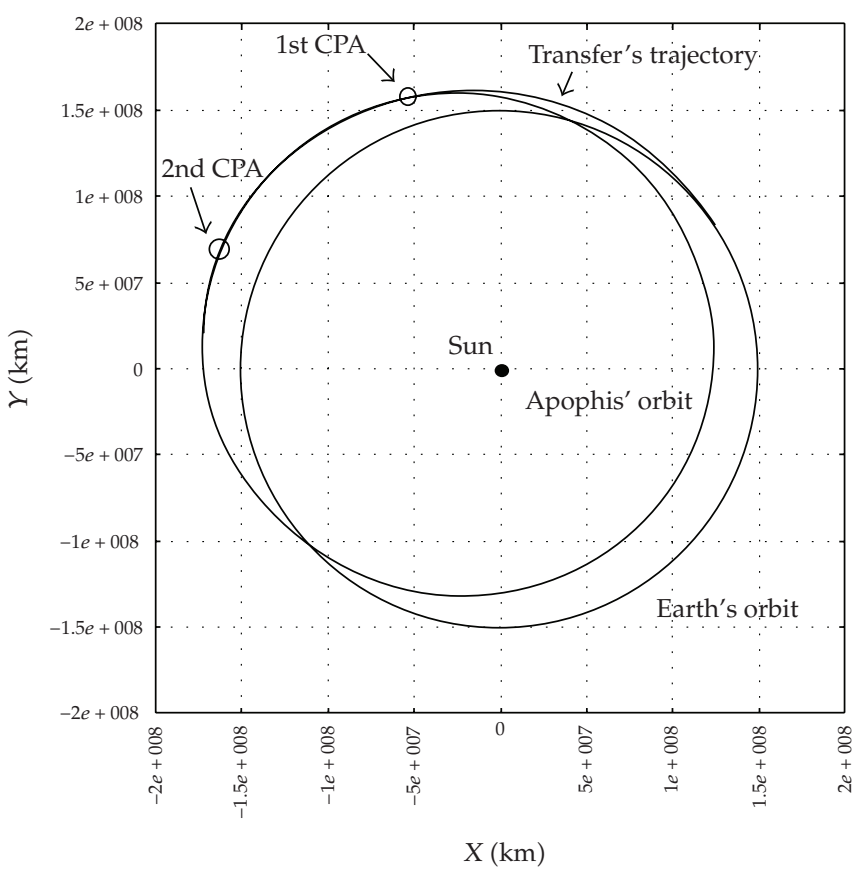

(a)

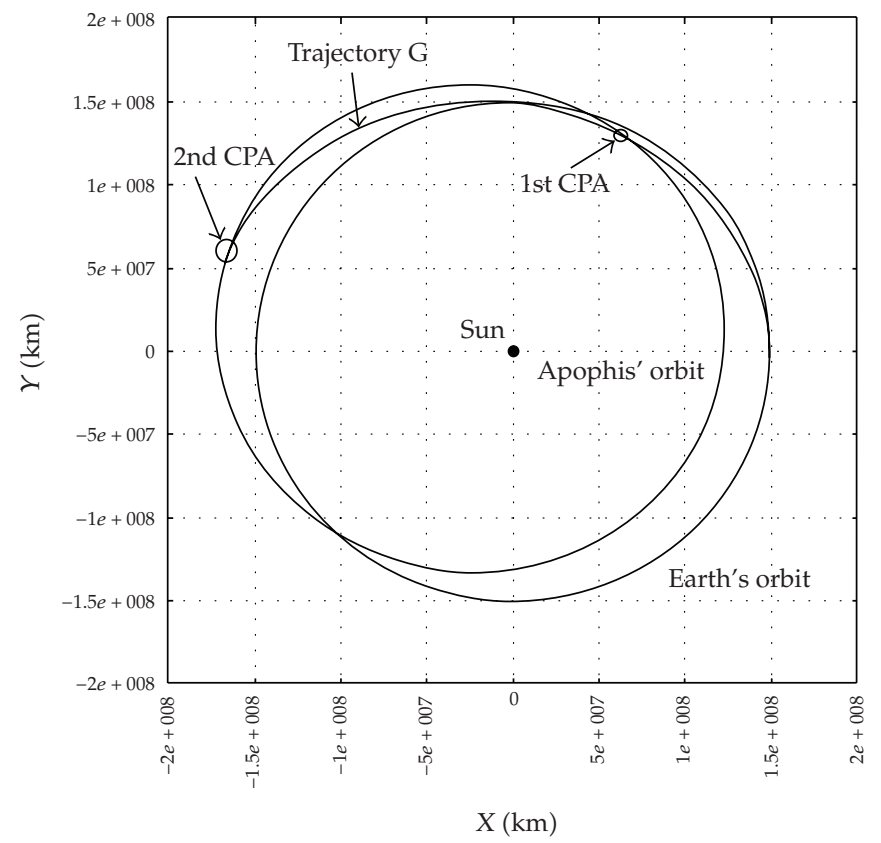

(b)

Figure 4: (a) Earth's and Apophis's orbits and the transfer trajectory found for Patched-conic transfer. (b) Earth's and Apophis's orbits and transfer trajectory G (heliocentric frame). 
The analysis of Tables 4 and 5 shows that the procedure described in Section 4 to generate transfer trajectories between the Earth and NEOs, derived from periodic orbits of Family $G$, requires $\Delta V \mathrm{~s}$ of $2 \%$ up to $4 \%$ less than the transfers conceived by Patchedconic approach. Besides, the spacecraft velocities relative to the asteroids in the CPAs, for the trajectories $G$, are the smallest. This can also provide fuel saving in occasional insertion maneuvers into an orbit around the asteroids. However, the smallest relative velocities always correspond to the longest transfer times.

\section{Conclusions}

In this work, a procedure capable of generating transfer trajectories between the Earth and the NEOs has been presented. These trajectories are derived from the periodic orbits around the Lagrangian point L1 and escape from the Earth-Moon system after a swing-by with the Moon.

In terms of $\Delta V_{\text {Total, }}$, those required by escape trajectories $\mathrm{G}$ are, in general, fewer than the ones required by conventional transfers (Patched-conic), between $2 \%$ up to $4 \%$. Besides, the spacecraft velocities relative to the asteroids are also, in general, less than those found by the conventional methods.

With regard to the transfer time, we verify that in two cases (Apophis and 1994 WR12) it was possible to find two CPAs. The time longest always corresponds to the smallest relative velocity in CPA for trajectories $\mathrm{G}$. We also verify that there are not any discrepancies between the transfer times of the two considered methods.

This way, we can conclude that the escape trajectories G presented in this work are a good alternative to design future missions destined to the NEOs.

Finally, future studies of techniques to accomplish a swing-by with the Earth and, thus, to gain more energy and a larger reach could provide the planning of transfers to more distant objects, including the internal planets.

\section{Acknowledgments}

The authors are grateful to the FAPESP (Fundação de Amparo à Pesquisa do Estado de São Paulo), processes 2005/05169-7 and 2006/04997-6, and the CNPq (Conselho Nacional para Desenvolvimento Científico e Tecnológico) Brazil, for the financial support.

\section{References}

[1] R. A. Broucke, "Periodic orbits in the restricted three-body problem with Earth-Moon masses," Tech. Rep. 32-1168, JPL, Los Angeles, Calif, USA, 1968.

[2] C. F. de Melo, E. E. N. Macau, O. C. Winter, and E. V. Neto, "Alternative paths for insertion of probes into high inclination lunar orbits," Advances in Space Research, vol. 40, no. 1, pp. 58-68, 2007.

[3] C. F. de Melo, E. E. N. Macau, and O. C. Winter, "Strategies for plane change of Earth orbits using lunar gravity and derived trajectories of family G," Celestial Mechanics E Dynamical Astronomy, vol. 103, no. 4, pp. 281-299, 2009.

[4] D. Lawden, "Perturbation maneuvers," Journal of the British Interplanetary Society, vol. 13, no. 5, 1954.

[5] M. A. Minovitch, "A method for determining interplanetary free-fall reconnaissance trajectories," Technical Memo TM-312-130, JPL, 1961.

[6] R. A. Broucke, "The celestial mechanics of gravity assist," in Proceedings of AIAA/AAS Astrodynamics Conference, Minneapolis, Minn, USA, August 1988. 
[7] C. E. Kohlhase and P. A. Penzo, "Voyager mission description," Space Science Reviews, vol. 21, no. 2, pp. 77-101, 1977.

[8] R. Carvell, “Ulysses-the Sun from above and below,” Space, vol. 1, pp. 18-55, 1985.

[9] D. Dunham and S. Davis, "Optimization of a multiple lunar swing-by trajectory sequence," Journal of the Astronautical Sciences, vol. 33, no. 3, pp. 275-288, 1985.

[10] C. Uphoff, "The art and science of lunar gravity assist, orbital mechanics and mission design," Advances in the Astronautical Sciences, vol. 69, pp. 333-346, 1989.

[11] A. F. B. A. Prado, "Orbital control of a satellite using the gravity of the moon," Journal of the Brazilian Society of Mechanical Sciences and Engineering, vol. 28, no. 1, pp. 105-110, 2006.

[12] R. Farquhar, "Detour a Comet, Journey of the International Cometary Explorer," Planetary Report, vol. 5, no. 3, pp. 4-6, 1985.

[13] C. D. Murray and S. F. Dermott, Solar System Dynamics, Cambridge University Press, Cambridge, Mass, USA, 1999.

[14] K. Deb, N. Padhye, and G. Neema, "Interplanetary trajectory optimization with swing-bys using evolutionary multi-objective optimization," in Proceedings of the 2nd International Symposium on Intelligence Computation and Applications (ISICA '07), L. Kang, Y. Liu, and S. Zeng, Eds., vol. 4683 of Lecture Notes in Computer Science, pp. 26-35, Wuhan, China, September 2007.

[15] E. E. N. Macau and C. Grebogi, "Control of chaos and its relevancy to spacecraft steering," Philosophical Transactions of the Royal Society of London. Series A, vol. 364, no. 1846, pp. 2463-2481, 2006.

[16] V. A. Chobotov, Orbital Mechanics, AIAA, 1991. 




Advances in

Operations Research

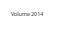

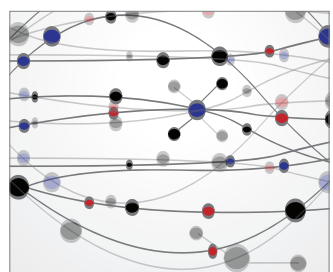

\section{The Scientific} World Journal
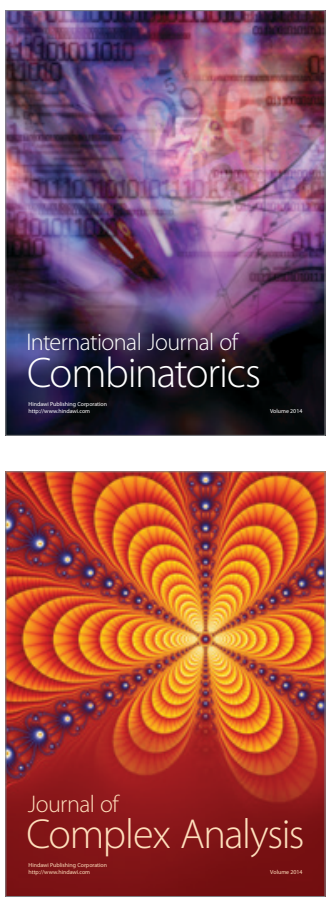

International Journal of

Mathematics and

Mathematical

Sciences
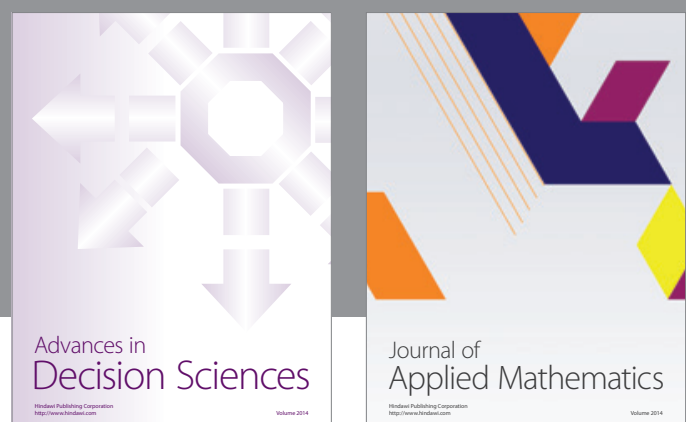

Journal of

Applied Mathematics
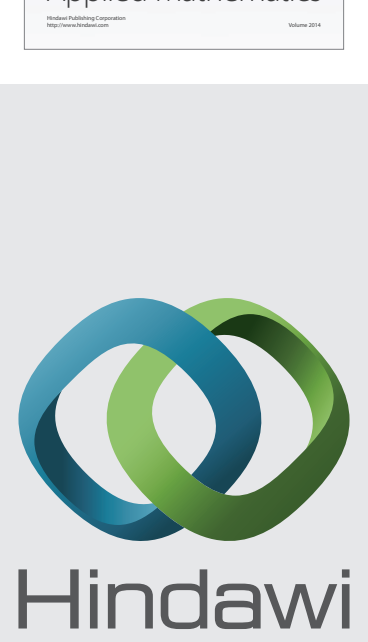

Submit your manuscripts at http://www.hindawi.com
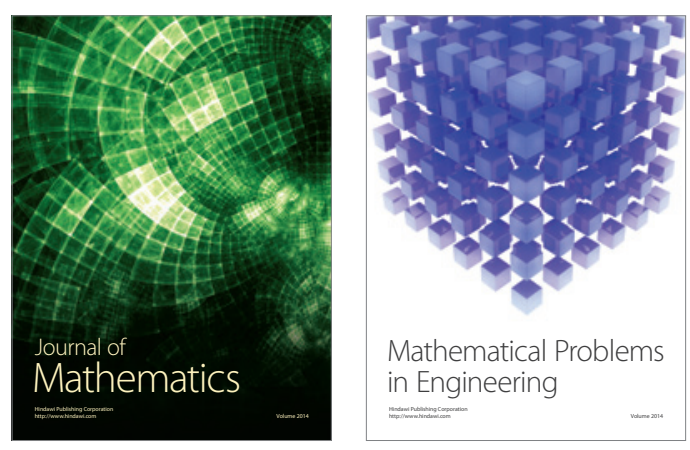

Mathematical Problems in Engineering
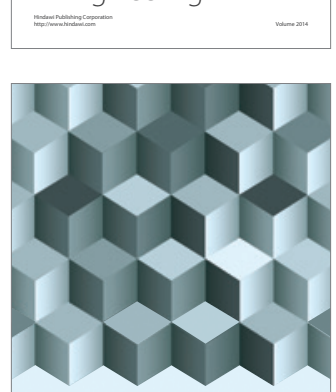

Journal of

Function Spaces
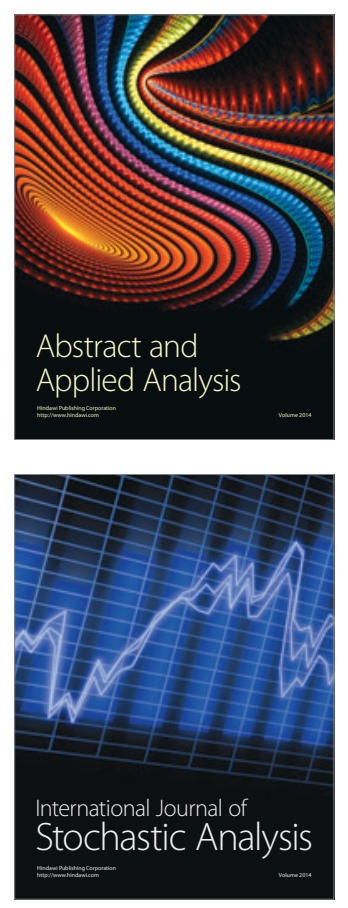

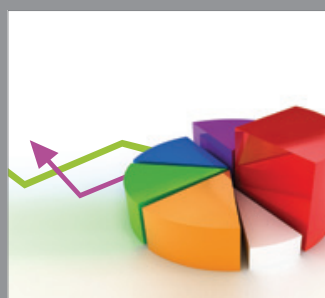

ournal of

Probability and Statistics

Promensencen
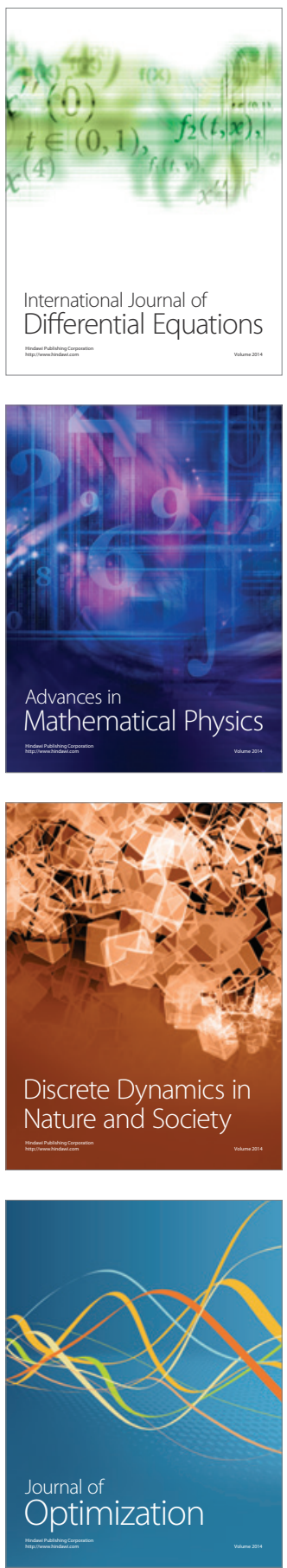